\title{
CONGRUENCES MODULO SQUARES OF PRIMES FOR FU'S $k$ DOTS BRACELET PARTITIONS
}

\author{
CRISTIAN-SILVIU RADU AND JAMES A. SELLERS \\ Dedicated to George Andrews on the occasion of his 75th birthday
}

\begin{abstract}
In 2007, Andrews and Paule introduced the family of functions $\Delta_{k}(n)$ which enumerate the number of broken $k$-diamond partitions for a fixed positive integer $k$. In that paper, Andrews and Paule proved that, for all $n \geq 0, \Delta_{1}(2 n+1) \equiv 0(\bmod 3)$ using a standard generating function argument. Soon after, Shishuo Fu provided a combinatorial proof of this same congruence. Fu also utilized this combinatorial approach to naturally define a generalization of broken $k$-diamond partitions which he called $k$ dots bracelet partitions. He denoted the number of $k$ dots bracelet partitions of $n$ by $\mathfrak{B}_{k}(n)$ and proved various congruence properties for these functions modulo primes and modulo powers of 2 . In this note, we extend the set of congruences proven by Fu by proving the following congruences:
\end{abstract} For all $n \geq 0$,

$$
\begin{aligned}
\mathfrak{B}_{5}(10 n+7) & \equiv 0 \quad\left(\bmod 5^{2}\right), \\
\mathfrak{B}_{7}(14 n+11) & \equiv 0 \quad\left(\bmod 7^{2}\right), \quad \text { and } \\
\mathfrak{B}_{11}(22 n+21) & \equiv 0 \quad\left(\bmod 11^{2}\right)
\end{aligned}
$$

We also conjecture an infinite family of congruences modulo powers of 7 which are satisfied by the function $\mathfrak{B}_{7}$.

\section{INTRODUCTION}

Broken $k$-diamond partitions were introduced in 2007 by Andrews and Paule [1]. These are constructed in such a way that the generating functions of their counting sequences $\left(\Delta_{k}(n)\right)_{n \geq 0}$ are closely related to modular forms. Namely,

$$
\begin{aligned}
\sum_{n=0}^{\infty} \Delta_{k}(n) q^{n} & =\prod_{n=1}^{\infty} \frac{\left(1-q^{2 n}\right)\left(1-q^{(2 k+1) n}\right)}{\left(1-q^{n}\right)^{3}\left(1-q^{(4 k+2) n}\right)} \\
& =q^{(k+1) / 12} \frac{\eta(2 \tau) \eta((2 k+1) \tau)}{\eta(\tau)^{3} \eta((4 k+2) \tau)}, \quad k \geq 1
\end{aligned}
$$

Date: August 20, 2012.

2010 Mathematics Subject Classification. Primary 11P83; Secondary 05A17.

Key words and phrases. broken $k$-diamonds, congruences, modular forms, partitions, $k$ dots bracelet partitions.

C. S. Radu was funded by the Austrian Science Fund (FWF), W1214-N15, project DK6 and by grant P2016-N18. The research was supported by the strategic program "Innovatives OÖ 2010 plus" by the Upper Austrian Government. 
where we recall the Dedekind eta function

$$
\eta(\tau):=q^{\frac{1}{24}} \prod_{n=1}^{\infty}\left(1-q^{n}\right) \quad\left(q=e^{2 \pi i \tau}\right) .
$$

In their original work, Andrews and Paule proved that, for all $n \geq 0$,

$$
\Delta_{1}(2 n+1) \equiv 0 \quad(\bmod 3)
$$

by utilizing generating function manipulations. Soon after, Hirschhorn and Sellers [4] reproved (1.2) by finding an explicit representation of the generating function for $\Delta_{1}(2 n+1)$ which implied (1.2), and Mortenson [5] developed a statistic on the partitions enumerated by $\Delta_{1}(2 n+1)$ which naturally breaks these partitions into three subsets of equal size (thus proving (1.2) combinatorially).

More recently, Shishuo Fu [2] proved (1.2) via a combinatorial argument as well. In the process, he generalized the notion of broken $k$-diamond partitions to combinatorial objects which he termed $k$ dots bracelet partitions. Fu [2] denoted the number of $k$ dots bracelet partitions of $n$ by $\mathfrak{B}_{k}(n)$. He then proved the following congruence properties satisfied by these functions (the first of which Fu termed "a natural generalization of" (1.2)).

Theorem 1.1. For $n \geq 0, k \geq 3$, if $k=p^{r}$ is a prime power, then

$$
\mathfrak{B}_{k}(2 n+1) \equiv 0 \quad(\bmod p)
$$

Theorem 1.2. For any $k \geq 3, s$ an integer between 1 and $p-1$ such that $12 s+1$ is a quadratic nonresidue modulo $p$, and any $n \geq 0$, if $p \mid k$ for some prime $p \geq 5$, then

$$
\mathfrak{B}_{k}(p n+s) \equiv 0 \quad(\bmod p)
$$

Theorem 1.3. For $n \geq 0, k \geq 3$ even, say $k=2^{m} l$, where $l$ is odd, we have

$$
\mathfrak{B}_{k}(2 n+1) \equiv 0 \quad\left(\bmod 2^{m}\right) .
$$

Our primary goal in this brief note is to prove the following theorem, thus extending the set of congruences mentioned above for $k$ dots bracelet partitions.

Theorem 1.4. For all $n \geq 0$,

$$
\begin{aligned}
& \mathfrak{B}_{5}(10 n+7) \equiv 0 \quad\left(\bmod 5^{2}\right), \\
& \mathfrak{B}_{7}(14 n+11) \equiv 0 \quad\left(\bmod 7^{2}\right), \text { and } \\
& \mathfrak{B}_{11}(22 n+21) \equiv 0\left(\bmod 11^{2}\right) \text {. }
\end{aligned}
$$

\section{Proof of Theorem 1.4}

For $p=5,7,11$, let

$$
F_{p}(\tau):=\eta(2 p \tau)^{12-p} \eta(2 \tau) \times\left(\frac{\eta^{p}(\tau)}{\eta(p \tau)}\right)^{p-1}
$$


We will see below that this is a natural choice since the generating function for $\mathfrak{B}_{p}(n)$ is given by

$$
\sum_{n=0}^{\infty} \mathcal{B}_{p}(n) q^{n}=\prod_{n=1}^{\infty} \frac{\left(1-q^{2 n}\right)\left(1-q^{p n}\right)}{\left(1-q^{n}\right)^{p}\left(1-q^{2 p n}\right)}
$$

We observe that

$$
F_{p}(\tau)=q^{p+\frac{1-p^{2}}{12}} \prod_{n=1}^{\infty}\left(1-q^{2 p n}\right)^{12-p}\left(1-q^{2 n}\right)\left(\frac{\left(1-q^{n}\right)^{p}}{1-q^{p n}}\right)^{p-1}
$$

Set

$$
\sum_{n=0}^{\infty} a_{p}(n) q^{n}:=\prod_{n=1}^{\infty}\left(1-q^{2 p n}\right)^{12-p}\left(1-q^{2 n}\right)\left(\frac{\left(1-q^{n}\right)^{p}}{1-q^{p n}}\right)^{p-1}
$$

Then

$$
\begin{aligned}
U_{2 p}\left(F_{p}(\tau)\right) & =U_{2 p}\left(q^{p+\frac{1-p^{2}}{12}} \sum_{n=0}^{\infty} a_{p}(n) q^{n}\right) \\
& =\sum_{n=0}^{\infty} a_{p}\left(2 p n+p+\frac{p^{2}-1}{12}\right)
\end{aligned}
$$

where $U_{2 p}$ is the "standard" $U$-operator [6, p. 28]. From Ono [6, Theorems 1.64 and 1.65] we find that $\left(\frac{\eta^{p}(\tau)}{\eta(p \tau)}\right)^{p-1}$ is a modular form for the group $\Gamma_{0}(p)$ of weight $(p-1)^{2} / 2$. Similarly, we find that $\eta(2 p \tau)^{12-p} \eta(2 \tau)$ is a modular form of weight $k_{p}:=(13-p) / 2$ with character $\chi_{p}(d):=\left(\frac{(-1)^{k} p}{d}\right)$ for the group $\Gamma_{0}(4 p)$. Consequently, $F_{p}(\tau)$ is a modular form of weight $w_{p}:=(13-p) / 2+(p-1)^{2} / 2$ and character $\chi_{p}(d)$ for the group $\Gamma_{0}(4 p)$. Then because of [6, Prop. 2.22] also $U_{2 p}\left(F_{p}(\tau)\right)$ is a modular form of weight $w_{p}$ and character $\chi_{p}(d)$ for the group $\Gamma_{0}(4 p)$. Using a variant of Sturm's theorem (see Ono [6, Theorem 2.58]) we find that

$$
\sum_{n=0}^{\infty} a_{p}\left(2 p n+p+\frac{p^{2}-1}{12}\right)=U_{2 p}\left(F_{p}(\tau)\right) \equiv 0 \quad\left(\bmod p^{2}\right)
$$

iff

$$
a_{p}\left(2 p n+p+\frac{p^{2}-1}{12}\right) \equiv 0 \quad\left(\bmod p^{2}\right)
$$

for the finite sequence of values $n=0,1, \ldots, \frac{w_{p}}{24}\left[\mathrm{SL}_{2}(\mathbb{Z}): \Gamma_{0}(4 p)\right]$. Using Ono $[6$, Proposition 1.7], we find that

$$
\frac{w_{p}}{24}\left[\mathrm{SL}_{2}(\mathbb{Z}): \Gamma_{0}(4 p)\right]=\frac{p+1}{8}\left(13-p+(p-1)^{2}\right) .
$$

We have verified that this finite set of congruences hold, and therefore

$$
U_{2 p}\left(F_{p}(\tau)\right) \equiv 0 \quad\left(\bmod p^{2}\right)
$$

for $p=5,7,11$.

Next note that

$$
U_{2 p}\left(F_{p}(\tau)\right) \equiv 0 \quad\left(\bmod p^{2}\right)
$$


implies that

$$
\prod_{n=1}^{\infty}\left(1-q^{n}\right)^{p-13} U_{2 p}\left(F_{p}(\tau)\right) \equiv 0 \quad\left(\bmod p^{2}\right)
$$

However,

$$
\prod_{n=1}^{\infty}\left(1-q^{n}\right)^{p-13} U_{2 p}\left(F_{p}(\tau)\right)=U_{2 p}\left(\prod_{n=1}^{\infty}\left(1-q^{2 p n}\right)^{p-13} F_{p}(\tau)\right)
$$

and

$$
U_{2 p}\left(\prod_{n=1}^{\infty}\left(1-q^{2 p n}\right)^{p-13} F_{p}(\tau)\right) \equiv U_{2 p}\left(\prod_{n=1}^{\infty}\left(1-q^{2 p n}\right)^{p-13} F_{p}(\tau)\left(\frac{\left(1-q^{n}\right)^{p}}{1-q^{p n}}\right)^{1-p}\right) \quad\left(\bmod p^{2}\right) .
$$

This implies that

$$
U_{2 p}\left(\prod_{n=1}^{\infty}\left(1-q^{2 p n}\right)^{p-13} F_{p}(\tau) \prod_{n=1}^{\infty}\left(\frac{\left(1-q^{n}\right)^{p}}{1-q^{p n}}\right)^{1-p}\right) \equiv 0 \quad\left(\bmod p^{2}\right) .
$$

From the definition of $F_{p}(\tau)$ we know

$$
\begin{aligned}
\prod_{n=1}^{\infty}\left(1-q^{2 p n}\right)^{p-13} F_{p}(\tau) \prod_{n=1}^{\infty}\left(\frac{\left(1-q^{n}\right)^{p}}{1-q^{p n}}\right)^{1-p} & =q^{p+\frac{1-p^{2}}{12}} \prod_{n=1}^{\infty} \frac{\left(1-q^{2 n}\right)\left(1-q^{p n}\right)}{\left(1-q^{n}\right)^{p}\left(1-q^{2 p n}\right)} \\
& =q^{p+\frac{1-p^{2}}{12}} \sum_{n=0}^{\infty} \mathcal{B}_{p}(n) q^{n} .
\end{aligned}
$$

Hence,

$$
U_{2 p}\left(q^{p+\frac{1-p^{2}}{12}} \sum_{n=0}^{\infty} \mathcal{B}_{p}(n) q^{n}\right)=\sum_{n=0}^{\infty} \mathcal{B}_{p}\left(2 p n+p+\frac{p^{2}-1}{12}\right) \equiv 0 \quad\left(\bmod p^{2}\right) .
$$

This completes the proof of Theorem 1.4.

\section{Concluding Remarks}

We close with two comments. First, given the combinatorial genesis of the definition of $\mathfrak{B}_{k}(n)$, it would be nice to have a combinatorial proof of Theorem 1.4. Secondly, we state the following conjectured infinite family of congruences:

Conjecture: For all $n \geq 0$ and all $\alpha \geq 1$,

$$
\mathfrak{B}_{7}\left(7^{\alpha} n+\lambda_{\alpha}\right) \equiv 0 \quad\left(\bmod 7^{\left\lceil\frac{\alpha-1}{2}\right\rceil}\right)
$$

where $\lambda_{\alpha}=\frac{1+7^{\alpha}}{2}$. This is an intriguing family of congruences given the similarity to the infinite family of congruences modulo powers of 7 which holds for the ordinary partition function $p(n)$ (as was originally proved by Watson [7] in 1938 and later proved in a more elementary fashion by Garvan [3]). 


\section{REFERENCES}

[1] G. E. Andrews and P. Paule. MacMahon's Partition Analysis XI: Broken Diamonds and Modular forms. Acta Arithmetica, 126:281-294, 2007.

[2] S. Fu. Combinatorial Proof of One Congruence for the Broken 1-Diamond Partition and a Generalization. Int. J. of Number Theory, 7(1):133-144, 2011.

[3] F. Garvan. A simple proof of watson's partition congruences for powers of 7. J. Austral. Math. Soc. Ser. A, 36(3):316-334, 1984.

[4] M. D. Hirschhorn and J. A. Sellers. On Recent Congruence Results of Andrews and Paule. Bulletin of the Australian Mathematical Society, 75:121-126, 2007.

[5] E. Mortenson. On the broken 1-diamond partition. Int. J. Number Theory, 4(2):199-218, 2008.

[6] K. Ono. The Web of Modularity: Arithmetic of the Coefficientsof the Modular Forms and q-series. Number 102 in CBMS Regional Conference Series in Mathematics. AMS, 2004.

[7] G. N. Watson. Ramanujan's Vermutung über Zerfällungsanzahlen. Journal für die Reine und Angewandte Mathematik, 179:97-128, 1938.

Research Institute for Symbolic Computation (RISC), Johannes Kepler University, A4040 Linz, Austria, SRAdu@RISC.Uni-LinZ.AC.AT

Department of Mathematics, Penn State University, University Park, PA 16802, USA, SELLERSJ@PSU.EDU 